\title{
Level of chronic life stress predicts clinical outcome in irritable bowel syndrome
}

\author{
E J Bennett, C C Tennant, C Piesse, C-A Badcock, J E Kellow
}

\begin{abstract}
Background-Life stress contributes to symptom onset and exacerbation in the majority of patients with irritable bowel syndrome (IBS) and functional dyspepsia (FD); research evidence is conflicting, however, as to the strength of these effects. Aims-To test prospectively the relation of chronic life stress threat to subsequent symptom intensity over time.

Patients-One hundred and seventeen consecutive outpatients satisfying the modified Rome criteria for IBS $(66 \%$ with one or more concurrent FD syndromes) participated.
\end{abstract}

Methods-The life stress and symptom intensity measures were determined from interview data collected independently at entry, and at six and 16 months; these measures assessed the potency of chronic life stress threat during the prior six months or more, and the severity and frequency of IBS and FD symptoms during the following two weeks.

Results-Chronic life stress threat was a powerful predictor of subsequent symptom intensity, explaining $97 \%$ of the variance on this measure over 16 months. No patient exposed to even one chronic highly threatening stressor improved clinically (by 50\%) over the 16 months; all patients who improved did so in the absence of such a stressor.

Conclusion-The level of chronic life stress threat predicts the clinical outcome in most patients with IBS/FD.

(Gut 1998;43:256-261)

Keywords: irritable bowel syndrome; chronic life stress threat; symptom intensity

Medicine, Royal North

Shore Hospital,

Sydney, Australia

E J Bennett

C Piesse

C-A Badcock

J E Kellow

Department of

Psychological

Medicine, Royal North

Shore Hospital,

Sydney, Australia

E J Bennett

C C Tennant

Correspondence to: Associate Prof. J E Kellow, Department of Medicine, Royal North Shore Hospital, St Leonards NSW 2065, Australia.

Accepted for publication 4 February 1998 tology, particularly in patients with IBS-FD syndromes. Despite these observations, the extent to which life stress contributes to the gastrointestinal, and extraintestinal symptoma- course of IBS and/or FD symptoms remains uncertain..$^{9} 10$

To extend our previous cross sectional findings, our aim was to examine, in patients with IBS, group and individual patterns of change in life stress and symptom intensity over time. Specifically we aimed to determine within subject: (1) covariance of life stress and subsequent symptom intensity over three time frames; (2) time lag relations (with and without relevant covariates); (3) the role of personality, age, sex, and emotional distress in the above; (4) the life stress predictors of any improvement or lack of improvement in symptom intensity over time; and (5) the life stress predictors of clinical ( $50 \%$ or more) improvement or no clinical improvement in symptom intensity over time. We hypothesised that: (1) life stress and subsequent symptom intensity will covary - that is, incremental increases/ decreases in symptom intensity will follow, and correspond with incremental increases/ decreases in life stress; (2) the presence or absence of severe chronic threat will precede major shifts in symptom intensity, or if present and unchanged, will inhibit clinical improvement; and (3) these effects will significantly exceed the effects of baseline symptomatology and personality on subsequent symptom intensity.

\section{Methods}

PATIENTS

The patient sample comprised 117 unselected outpatients ( 31 men, 86 women; aged 43 (12) years) with well defined IBS who satisfied the inclusion criteria - that is, fulfilment of the criteria of Drossman ${ }^{11}$; absence of organic disease by appropriate investigations; age less than 70 years; and no difficulty conversing in English. This extension of a cross sectional study ${ }^{8}$ includes the subgroup of participants with IBS, $66 \%$ of whom were also diagnosed with one or more FD syndromes. Classification of FGID was undertaken as previously. ${ }^{8}$ The protocol was approved by the Medical Research Ethics Committee of the Royal North Shore Hospital, and all patients gave written informed consent.

EXPERIMENTAL PROTOCOL

All patients underwent a detailed semistructured interview and completed self report psychological and symptom questionnaires; psychosocial and symptom data were collected in a blind and independent manner. Figure 1 shows the longitudinal study design. Life stress and symptom intensity were assessed separately by two independent interviewers at entry, at six months, and at 16 months; life stress was 


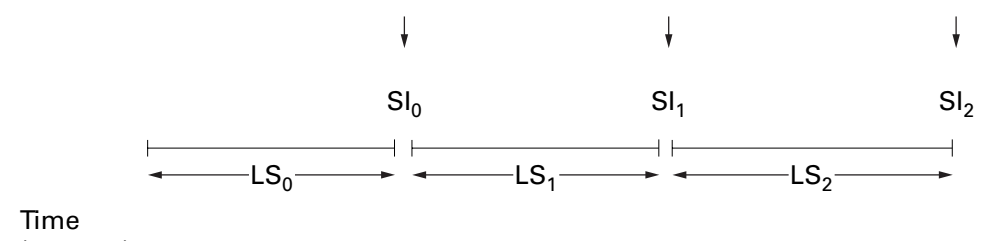

(months) -6

0

6

16

Figure 1 Schematic illustration of the longitudinal study design. LS, average chronic threat intensity during the six months or more prior to entry $\left(L S_{0}\right)$, at six months $\left(L S_{\nu}\right)$, and 16 months $\left(L S_{2}\right) ; S I$, average symptom intensity during the following two weeks $\left(S_{o}\right.$, $\left.S I_{1}, S I_{2}\right)$.

assessed two weeks prior to symptom intensity. The time period between assessments of life stress permitted at least six months for each chronic stressor assessment. Psychological questionnaires to assess personality and anxiety were administered at entry only. The depression scale was administered at entry and at 16 months and classification into diagnostic category was repeated at 16 months.

\section{MEASURES}

Symptom intensity

The intensity of current IBS and FD symptoms was defined as the product of the frequency and the severity of each IBS/FD symptom during the previous two weeks, each dimension being graded on a four point scale. Frequency was scored as: $0=$ not a problem; $1=$ once only in the two week period; $2=$ occurred more than once each week; and $3=$ occurred almost every day, or daily. Severity was scored as follows: $0=$ this symptom had not occurred; $1=$ mild severity (no remedy sought, no remedy tried); 2 $=$ moderate severity (remedy sought, but daily activities were not affected); and $3=$ severe (interfering with daily activities). As well as symptom intensity assessments at entry, six months, and 16 months, the duration of baseline symptom intensity was also noted-that is, the number of months that symptom intensity was at entry level prior to initial assessment.

Life stressors

The semistructured interview procedure of Brown and Harris, ${ }^{12}$ the Life Events and Difficulties Schedule (LEDS), was used. Only chronic stressors (of at least six months duration) were included in the analyses; stressors of less than six months duration were excluded. Chronic stressor situations included divorce, relationship difficulties, serious illness (of self or other), lawsuits, business failures, housing difficulties, and forced redundancies; other difficulties arose within the context of caring for a family member with significant physical and/or emotional problems. Each chronic stressor rated as severe and likely to be independent of IBS/FD symptoms and psychiatric illness was included, and each was separately rated for threat and for goal frustration on a four point scale ranging from $0=$ mild to 3 = very severe; each stressor rating represented the average severity of threat/goal frustration for at least six months prior to each assessment (see Bennett and colleagues ${ }^{8}$ for further details). Measures of chronic threat were both dichotomous (the presence or absence of at least one chronic stressor that was highly threatening), and continuous (a "total chronic threat" score-namely, the sum of the chronic threat ratings across all chronic stressors). Similar variables were derived from stressor ratings of chronic goal frustration. Clinical validity and reliability have been established for the LEDS procedure ${ }^{13}$ and inter-rater reliability is high. ${ }^{13}$

\section{Psychological measures}

Standardised questionnaires assessed specific dimensions of emotional distress/current mood state (depression ${ }^{14}$ and state anxiety ${ }^{15}$ ), and of personality (neuroticism and extraversion-introversion, ${ }^{16}$ trait anxiety, ${ }^{15}$ trait anger, ${ }^{17}$ and general hypochondriasis ${ }^{18}$ ).

\section{STATISTICAL ANALYSES}

\section{Group patterns of change over time}

From symptom intensity and life stress total chronic threat and total goal frustration measures (assessed at entry, and at six and 16 months), group means, and standard deviations were calculated.

\section{Within subject change over time}

To assess the covariance of life stress and symptom intensity over time, global repeated measures of analyses of variance were performed. In the first, the changes in symptom intensity scores over time were tested; in the second, these changes were assessed with life stress scores as covariates. The extent to which changes in life stress accounted for changes in symptom intensity scores, was assessed by considering the significance and the magnitude of the changes in symptom intensity with and without the life stress covariates (using the Time ${ }_{\mathrm{ws}}$ Effect size measure, $\Omega^{2}$ ).

\section{Time lagged relation of life stress to symptom} intensity

To determine the time lagged relation of life stress (and emotional distress) to subsequent symptom intensity, and to identify potential mediators of the life stress-symptom intensity relation, linear regression analysis and/or analysis of variance was performed (with or without relevant covariates). Logistic regression analysis was used to determine the life stress predictors of improvement versus lack of improvement in symptom intensity over the whole follow up period. Analyses controlled for the effects of baseline symptom intensity and the duration of symptom intensity at that level prior to entry; other covariates (demographic, emotional distress, and personality) were included when relevant because of their potential to confound with measures of life stress and symptom intensity, and their association over time. To compare the effects of each independent variable on symptom intensity outcome after removal of the confounding effects of all variables in the model, partial correlation values (partial $\eta^{2}$ ) were calculated. 
Table 1 Proportion of patients with change (worse, improved) or no change (same) in symptom intensity and life stressor chronic threat, and/or chronic goal frustration) over 16 months

\begin{tabular}{|c|c|c|c|}
\hline Parameter of change & $\begin{array}{l}\text { Worse } \\
(\%)\end{array}$ & $\begin{array}{l}\text { Same } \\
(\%)\end{array}$ & $\begin{array}{l}\text { Improved } \\
(\%)\end{array}$ \\
\hline \multicolumn{4}{|l|}{ Symptom intensity } \\
\hline Entry to 6 months & 9 & 13 & 78 \\
\hline Entry to 16 months & 9 & 12 & 79 \\
\hline 6 to 16 months & 21 & 33 & 45 \\
\hline \multicolumn{4}{|l|}{ Life stressor } \\
\hline \multicolumn{4}{|l|}{ Chronic threat } \\
\hline Entry to 6 months & 1 & 3 & 96 \\
\hline Entry to 16 months & 0 & 3 & 97 \\
\hline 6 to 16 months & 16 & 51 & 33 \\
\hline \multicolumn{4}{|c|}{ Chronic goal frustration } \\
\hline Entry to 6 months & 1 & 2 & 97 \\
\hline Entry to 16 months & 0 & 3 & 97 \\
\hline 6 to 16 months & 18 & 59 & 23 \\
\hline
\end{tabular}

Table 2 FGID diagnosis at 16 months

\begin{tabular}{lll}
\hline FGID diagnosis at 16 months & $\begin{array}{l}\text { No of } \\
\text { subjects }\end{array}$ & $\begin{array}{l}\text { Sample } \\
\%\end{array}$ \\
\hline IBS & 74 & 63 \\
Unspecified functional bowel disorder & 28 & 24 \\
Functional conspitation & 3 & 2 \\
Subclinical symptoms & 9 & 8 \\
Asymptomatic for more than 6 months & 3 & 3 \\
Total & 117 & 100 \\
IBS + one or more functional dyspepsia & & \\
$\quad$ syndromes & 45 & 38 \\
\hline
\end{tabular}

IBS, irritable bowel syndrome.

\section{Results}

GROUP AND INDIVIDUAL PATTERNS OF CHANGE IN LIFE STRESS AND SYMPTOM INTENSITY OVER TIME Group mean scores reduced over the follow up period for life stress (total chronic threat and total chronic goal frustration) and for symptom intensity assessed at entry, and at six and 16 months (figs 2 and 3 respectively). Table 1 shows that although group patterns of change for both sysmptom intensity and life stress were in the direction of a general improvement for most patients from entry to six months, and from entry to 16 months, symptom intensity and/or life stress either worsened or remained the same for most participants from six to 16 months. That is, the direction of change became increasingly variable during the follow up period consistent with a pattern of change that was independent of systematic extraneous influences. Diagnostic status at 16 months (table 2) reveals that although the proportion of patients with a diagnosis of IBS and/or with concomitant FD syndromes reduced over time, only $11 \%$ had subclinical or no symptoms at 16 months.

WITHIN SUBJECT RELATION OF LIFE STRESS TO SUBSEQUENT SYMPTOM INTENSITY OVER TIME Covariance over three time frames

Taking into account for each individual all changes over time in symptom intensity and chronic threat analysis revealed a high degree of covariance of life stress and symptom intensity scores. Almost all (97\%) of the variance in symptom index over time apportioned to Time $_{\mathrm{ws}}$ effects $\left(\Omega^{2}=0.42 ; F_{2,232}=86 ; \mathrm{p}<0.0001\right)$, was accounted for by quantitative changes in one component of life stress - chronic threat. In the presence of chronic threat, chronic goal frustration did not contribute significantly to

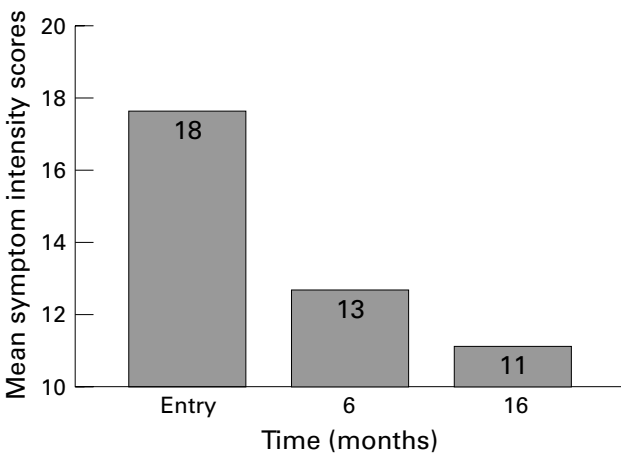

Figure 3 Mean symptom intensity scores at entry, six months, and 16 months.

changes in symptom index over time-hence, in all subsequent analyses only the chronic threat component of life stress was included.

\section{Time lag relations (with and without relevant} covariates)

Life stress during the first six months of the follow up period was highly predictive of symptom intensity at 16 months. The presence of one or more highly threatening chronic difficulties (from entry to six months) contributed significantly to this long term prediction of symptom intensity $\left(F_{1,115}=18.4 ; \mathrm{p}<0.0001\right)$. This relation remained significant even after controlling for the effects of baseline symptomatology (symptom intensity at entry, and duration at that level of intensity prior to entry), emotional distress (anxiety, depression), age, and sex $\left(F_{7,109}=12 ; \mathrm{p}<0.0001\right)$. Post hoc analyses determined: (1) the direction of effect to be firmly in the direction of life stress predicting subsequent symptom intensity, and not the reverse; and (2) the relation of proximal life stress (during the previous six months or more) to exceed distal life stress significantly (more than 10 months previously) as a predictor of symptom intensity at 16 months; partial $\eta^{2}$ values were 0.432 and 0.127 respectively.

Role of personality, age, sex, and emotional distress No personality characteristic (trait anxiety, neuroticism, extroversion, trait anger, general hypochondriasis), emotional state (anxiety and depression), or age or sex variable contributed to symptom intensity at 16 months or altered the strength of the relation of life stress to symptom intensity over time. Only in the absence of life stress variables did any emotional

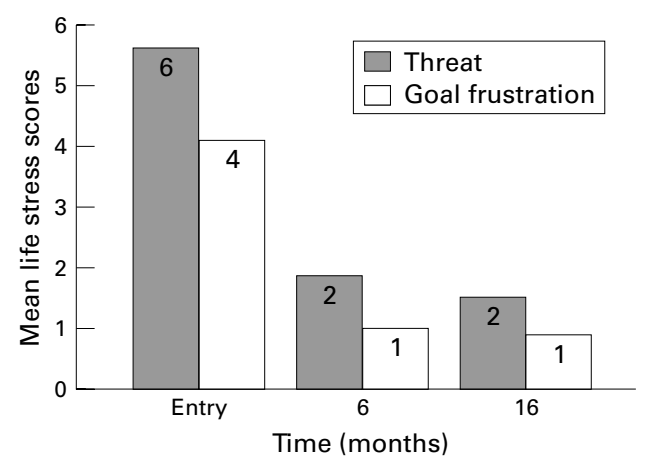

Figure 2 Mean chronic life stress scores for threat and goal frustration at entry, six months, and 16 months. 
Table 3 Clinical improvement as a function of the presence or absence of one or more highly threatening chronic difficulties during the previous 10 months

One or more highly threatening chronic difficulty

\begin{tabular}{lll} 
Clinical improvement & Absent $(\%) \dagger$ & Present $(\%) \neq$ \\
\hline Absent & 24 & 35 \\
Present & 41 & 0
\end{tabular}

${ }^{\star}$ Defined as the presence or absence of a $50 \%$ or more improvement in symptom intensity at 16 months.

†In the cohort without highly threatening chronic difficulties, the relative risk of no clinical improvement $v$ clinical improvement was 0.4 (95\% CI 0.3 to 0.5$)$.

$\ddagger$ In the cohort with highly threatening chronic difficulties, the relative risk of no clinical improvement $v$ clinical improvement could not be calculated as none of the patients exposed to this stressor improved by $50 \%$ or more.

distress variable (specifically anxiety state at entry) predict symptom intensity at six months $\left(F_{3,113}=37.0 ; \mathrm{p}<0.0001\right)$ and this effect was modest (partial $\eta^{2}=0.079$ ).

Life stress predictors of any improvement or lack of improvement in symptom intensity over 16 months Failure to improve symptomatically over 16 months was strongly predicted by the presence of at least one highly threatening chronic difficulty during the final 10 months (or to a lesser extent during the first six months) of the follow up period, with a specificity of $85 \%$, a sensitivity of $85 \%$, and an odds ratio of $33: 1(95 \%$ confidence interval 17 to 94 ). Controlling for emotional distress, age, and sex did not alter this relation.

Life stress predictors of clinical improvement or no clinical improvement over 16 months

Table 3 categorises clinical (50\% or more) improvement in symptom intensity by the presence of a significant chronic stressor. No patient exposed to one or more such stressor during the final 10 months of the follow up period (35\% of sample) improved by $50 \%$ or more (and conversely, all individuals who improved clinically $(41 \%)$, did so in the absence of this stressor). This precise differentiation was true for the majority $(76 \%)$ of patients. For $24 \%$ of patients, this chronic stressor (also personality, mood state, age, and sex) failed to explain the absence of a clinical improvement. Table 3 presents the relative risk estimates for the cohorts chronic stressor present and chronic stressor absent.

\section{Discussion}

This longitudinal study is the first, we believe, to assess the relation of chronic and severe life stress to subsequent symptom intensity in patients with IBS (with and without concomitant FD). The results overwhelmingly support the premise that in patients with these functional gastrointestinal disorders a component of life stress - severe and chronic threathas large and consistent effects on symptom intensity over time. New information includes evidence that: (1) almost all of the variance within individuals in symptom intensity levels (assessed at three time points over 16 months) was explained by the severity of chronic threat during the prior six months or more; and (2) the presence of one or more highly threatening chronic difficulties (over and above the effects of baseline symptomatology) strongly predicted high levels of symptom intensity even after a time lag of 10 months, while continuing high levels of life stress from six to 16 months, significantly reduced the likelihood of any improvement in symptom intensity over the entire 16 months. Indeed, no patient exposed to even one such stressor improved clinically (by at least $50 \%$ ) over the follow up period. The fact that the temporal relation was unequivocally as hypothesised-that is, life stress predicted subsequent symptom intensity (and not the reverse), and also that the effects were highly significant over and above that expected from symptoms alone and were uninfluenced by systematic extraneous factors, personality, or mood state, fully supports early reports ${ }^{4}$ that life stress has powerful influences on the course of the disorder for the majority of patients with IBS.

The research design took into account the chronic, fluctuating, and recurrent course of IBS, ${ }^{19-22}$ the frequent concurrence of FD syndromes with IBS, ${ }^{20} 22$ and the minimum duration of a "chronic" stressor situation (six months or more), while the selection of the chronic (life stress) threat as the type of life stress most likely to provoke significant long term changes in IBS/FD was based on our recent findings of a significant unmediated relation between number of FD/IBS syndromes present and the intensity of recent chronic (life stress) threat. ${ }^{8}$ Furthermore, the study sample was large and the methodology ensured reliable diagnoses, objective and independent life stress and symptom intensity assessments, and a sophisticated approach to the analysis of their inter-relation over time (for example, by statistically controlling for initial conditions). LS ratings were also independent of psychological and gut influences. The rater was blind to personal perceptions such as how individuals felt or how they reacted to particular situations, and stressors confounded by IBS/FD symptomatology, or its life stress consequences, were not included. The failure of psychological variables (for example, neuroticism, depression) to mediate the relation of life stress to symptom intensity over time or to predict subsequent life stress or symptom intensity significantly, statistically confirms that these life stress ratings are free of contamination from psychological influences. The independence of life stress ratings, and the wide range of responses on all measures both within and between subjects over time indicates that the IBS/FD cohort identified for this study was ideally suited to the evaluation of the relative effects of stressful chronic situations versus stress proneness (personality style) and illness related (hypochondriacal) attitudes on subsequent symptom intensity. It is important to note, however, that the findings of this study cannot be generalised to community (nonpatient) IBS populations or to patients with more complex medical histories.

Several core elements of the current findings are of particular interest - the overall strength 
and consistency of the relation, the type of stressor that seems to provoke major changes in functional gut symptomatology, and the stressor threshold that seems to predict so precisely clinical outcome for most IBS patients. The superiority of chronic highly threatening stressors over all other potential predictors in this study (age, sex, emotional distress, personality, and illness related attitudes) suggests that the experience of the stressor, and efforts (cognitive and behavioural) to deal with the stressor situation, constitute the primary link between prolonged exposure to threat and changes in symptom intensity. The chronic stressor in this study is similar to the major life stress situations that seemed to provoke or aggravate IBS/FD (or by their absence to relieve IBS/FD symptoms) both in early studies, ${ }^{419}$ and in more recent studies ${ }^{128}$ using more formal methods - that is, the LEDS. ${ }^{12}$ The relation of these chronic stressors (previously unexplored in IBS over time) to IBS/FD symptomatology is stronger than the presence of negative (usually transient) life events which have either a small effect, ${ }^{23-26}$ or no effect, ${ }^{27-29}$ and are stronger than the presence of daily irritations or the most bothersome events of the day, ${ }^{24} 3031$ which also have no effect.

Stressor threshold adds an important nonlinear dimension to the relation of life stress to subsequent symptom intensity. Dichotomous life stress predictors divided the sample into two subgroups of patients irrespective of their initial symptomatology: the majority $(76 \%)$, where the clinical outcome was highly sensitive to a specific threshold for stress (the presence/ absence of one or more chronic highly threatening stressor); and the remainder $(24 \%)$, where failure to improve (at lower levels of stress) could not be explained by the psychological dimensions assessed in this study. This does not exclude the possibility that idiosyncratic effects from any of a number of biogenetic and/or cognitive-behavioural factors may have inhibited improvement, and/or the perception of improvement for some individuals. It is also possible that differences in management strategies, which were not assessed in this study, might help to explain differences in clinical outcome; however, it seems reasonable to expect that clinical management protocols would be most effective early in the follow up period. From a design perspective, the findings of this study highlight the importance of considering baseline conditions and non-linear (threshold) as well as linear effects; they are also consistent with concepts of dynamic interplay within a biopsychosocial framework ${ }^{32}$ and the likelihood of diverse outcomes from even small differences in initial conditions. ${ }^{33}$

The findings from this study are relevant to the clinical management of patients with IBS. Evidence that the presence of even one highly threatening chronic stressor inhibits improvement, while its reduction or absence may be a prerequisite for a significant improvement, sets clear goals for stress reduction management. Thus, whether the patient is exposed to one, or multiple such stressors, the therapeutic goal would be to reduce each stressor to below the high threat threshold and to maintain threat at a more manageable level over time and changing circumstances. Clearly the sustained success of psychologically based treatment programmes over reassurance, placebo, and drug treatment, ${ }^{5-7}$ suggests that this may be a highly achievable goal.

In conclusion, this study incorporated novel design features to assess the relation of chronic life stress to subsequent symptom intensity in a large group of patients with IBS over three consecutive time periods. The results suggest that for most subjects: (1) chronic highly threatening stressors significantly influence subsequent symptom intensity; (2) these effects exceed the effects of baseline gut symptomatology; and (3) the relation is strong, consistent, and unequivocally in one direction; is uninfluenced by personality, age, sex, anxiety, and depression; and is largely independent of systematic exogenous influences. For the majority of patients with IBS, the results also suggest a particular threshold for chronic threat, above and below which one can predict the absence or the presence of a clinical improvement over time, thereby providing a workable goal for stress management regimens.

The help and support of members of the Departments of Gastroenterology and Psychological Medicine, Royal North Shore Hospital, and that of Ms Kathryn Palmer for rating the chronic stressors, and Dr Alan Taylor of Macquarie University, Sydney, for his advice with respect to statistical analyses, is most gratefully acknowledged.

1 Craig TK, Brown GW. Goal frustration and life events in the tiology of painful gastrointestinal disorder. $\mathcal{F}$ Psychosom Res 1984;28:411-21.

2 Bennett EJ, Beaurepaire J, Langeluddecke P, et al. Life stress and non-ulcer dyspepsia: a case-control study. $\mathcal{F}$ Psychosom Res 1991;35:579-90.

3 Ford MJ. The irritable bowel syndrome. 7 Psychosom Res 1986;30:399-410.

4 Chaudhary NA, Truelove SC. The irritable colon syndrome: a study of the clinical features, predisposing causes, and prognosis in 130 cases. Q F Med 1962;31:307-23.

5 Guthrie E, Creed F, Dawson D, et al. A controlled trial of Guthrie E, Creed F, Dawson D, et al. A controlled trial of
psychological treatment for the irritable bowel syndrome. psychological treatment for the it

6 Shaw G, Srivastava ED, Sadlier M, et al. Stress management for irritable bowel syndrome: a controlled study. Digestion 1991;50:36-42.

7 Rumsay N. Group stress management programmes v pharmacological treatment in the treatment of the irritable bowel syndrome. In Heaton KW, Creed F, Goeting NIM, eds. Towards confident management of irritable bowel syndrome. Lyme Regis: Duphar, 1991:33-9.

8 Bennett EJ, Piesse C, Palmer K, et al. Functional gastrointestinal disorders: psychological, social, and somatic features. Gut 1998;42:414-20.

9 Drossman DA. Psychosocial aspects of the functional gastrointestinal disorders. In Corazziari E, ed. Neurogastroenterology. Berlin, New York: de Gruyter, 1996.

10 Whitehead WE. Assessing the effects of stress on physical symptoms. Health Psychol 1994;13:99-102.

11 Drossman DA. The functional gastrointestinal disorders: diagnosis, pathophysiology, and treatment. Boston: Little, diagnosis, pathophysio.

12 Brown GW, Harris TO. Social origins of depression: a study of psychiatric disorder in women. London: Tavistock, 1978.

13 Tennant C, Smith A, Bebbington P, et al. The contextual threat of life events: the concept and its reliability. Psychol Med 1979;9:525-8.

4 Radloff LS. The CES-D scale: a self-report depression scale for research in the general population. Applied Psychological Measurement 1977;1:385-401.

15 Spielberger CD, Gorsuch RL, Lushene R, et al. Manual for the State-Trait Anxiety Inventory STAI (Form Y). Palo Alto: Consulting Psych Press, 1983.

16 Grayson D. A latent trait analysis of Eysenck Personality Questionnaire. F Psychiatr Res 1986;20:217-35.

17 Spielberger CD, Jacobs G, Russell S, et al. Assessment of anger: the State-Trait Anger Scale. In: Butcher JN, anger: the Statberger CP, eds. Advances in personality assessment. Vol Spielberger CP, eds. Advances
2. Hillsdale, NY: LEA, 1983.

18 Pilowsky I, Spence ND. Manual for the illness behaviour questionnaire (IBQ). 2nd edn. Adelaide: University of Adelaide, 1983. 
19 Waller SL, Misiewicz JJ. Prognosis in the irritable-bowel syndrome. Lancet 1969;ii:754-6.

20 Talley NJ, Weaver AL, Zinsmeister AR, et al. Onset and disappearance of gastrointestinal symptoms and functional gastrointestinal disorders. Am f Epidemiol 1992;136:16576.

21 Locke GR III, Talley NJ, Zinsmeister AR, et al. Evidence that the irritable bowel syndrome and functional dyspepsi are a single disorder: a longitudinal population-based study [abstract]. Gastroenterology 1996;110:A26.

22 Agréus L, Svärdsudd K, Nyrén O, et al. Irritable bowel syndrome and dyspepsia in the general population: overlap and lack of stability over time. Gastroenterology 1995;109: 671-80.

23 Whitehead WE, Crowell MD, Robinson JC, et al. Effects of stressful life events on bowel symptoms: subjects with irritable bowel syndrome compared with subjects without bowel dysfunction. Gut 1992;33:825-30.

24 Hui WM, Shiu LP, Lam SK. The perception of life events and daily stress in nonulcer dyspepsia. Am $\mathcal{F}$ Gastroenterol 1991;86:292-6.

25 Dinan TG, O'Keane V, O'Boyle C, et al. A comparison of the mental status, personality profiles and life events of patients with irritable bowel syndrome and peptic ulcer disease. Acta Psychiatr Scand 1991;84:26-8.
26 Haug TT, Wilhelmsen I, Berstad A, et al. Life events and stress in patients with dyspepsia compared with patients with duodenal ulcer and healthy controls. Scand $f$ Gastroenterol 1995;30:524-30.

27 Talley NJ, Piper DW. Major life event stress and dyspepsia of unknown cause: a case control study. Gut 1986;27:12-34.

28 Talley NJ, Piper DW. A prospective study of social factors and major life event stress in patients with dyspepsia of unknown cause. Scand $\mathcal{F}$ Gastroenterol 1987;22:268-72.

29 Drossman DA, McKee, DC, Sandler R, et al. Psychosocial factors in the irritable bowel syndrome: a multivariate study of patients and nonpatients with irritable bowel syndrome. Gastroenterology 1988;95:701-8.

30 Suls J, Wan CK, Blanchard EB. A multilevel data-analytic approach for evaluation of relationships between daily life stressors and symptomatology: patients with irritable bowel syndrome. Health Psychol 1994;13:103-13.

31 Dancey CP, Whitehouse A, Painter J, et al. The relationship between hassles, uplifts and irritable bowel syndrome: a preliminary study. $\mathcal{F}$ Psychosom Res 1995;7:827-32.

32 Cassileth LR, Drossman DA. Psychosocial factors in gastrointestinal illness. Psychother Psychosom 1993;59:131-43.

33 Mandel DR. Chaos theory, sensitive dependence, and the logistic equation. Am Psychol 1995;50:106-7. 